\title{
Expression of retinoid-related genes in serum-free cultures of normal, immortalized and malignant human oral keratinocytes
}

\author{
DIRK DRESSLER ${ }^{1,2 *}$, ZSOLT SARANG $^{1 *}$, ZSUZSA SZONDY $^{3}$, \\ KARIN ENGELHART ${ }^{2}$ and ROLAND C. GRAFSTRÖM ${ }^{1}$ \\ ${ }^{1}$ Institute of Environmental Medicine, Karolinska Institutet, S-171 77 Stockholm, Sweden; ${ }^{2}$ BioTeSys GmbH, \\ D-73728 Esslingen, Germany; ${ }^{3}$ Department of Biochemistry and Molecular Biology, \\ University of Debrecen, H-4012 Debrecen, Hungary
}

Received January 3, 2002; Accepted February 12, 2002

\begin{abstract}
Retinoids are used in the clinical treatment of oral squamous carcinoma, including both early and late stages. Inter-individual variation in responsiveness, including a common insensitivity of advanced stages, suggest that changes in retinoid-related functions might characterize tumor development. To investigate a genetic basis for this hypothesis, an in vitro multi-step model of carcinogenesis involving normal (NOK), SV40 T antigen-immortalized (SVpgC2a) and malignant ( $\mathrm{SqCC} / \mathrm{Y} 1)$ oral keratinocytes was analysed under identical culture conditions using micro-array technique (Affymetrix HG_U95A chip) for expression of 52 genes related to retinoid metabolism and actions. The variable detection of between 22-26 transcripts in the cell lines, involving binding/transport factors, receptors, transcriptional activators/repressors and responsive genes, indicated specificity in regards to the expression of known retinoid-related genes in oral keratinocytes. The transformed cell lines variably exhibited differences as compared to NOK, i.e., lower transcript levels for cellular retinol binding protein, the cellular retinoic acid binding protein II (CRABP II) and retinoic acid receptor $\gamma$, whereas in contrast, the levels of CRABP I were higher. Transcripts for proteins interacting with nuclear retinoid receptors were similarly expressed among the cell types, whereas transcripts for retinoid-metabolizing enzymes were generally not detected. Finally, transcripts of retinoid-responsive genes, including RARRES3, RI58, NN8-4AG and midkine, were variably expressed. The
\end{abstract}

Correspondence to: Dr Roland C. Grafström, Experimental Carcinogenesis, Institute of Environmental Medicine, Karolinska Institutet, S-171 77 Stockholm, Sweden

E-mail: roland.grafstrom@imm.ki.se

*Contributed equally

Key words: human oral keratinocytes, retinoid, malignant transformation, serum-free culture overall results imply selective expression of retinoid-related functions in normal and transformed keratinocytes, and that cell transformation can impair the capacity for binding and storage of retinol as well as retinoic acid-mediated signalling. These multiple alterations are consistent with possible retinoid insensitivity during oral carcinogenesis.

\section{Introduction}

Vitamin A and some of its analogues (retinoids) are established modulators of epithelial cell differentiation in vivo and in vitro $(1,2)$. One of the major physiological functions of vitamin A may be to prevent keratinization of non-keratinizing epithelia $(1,3)$. Squamous metaplasia, which develops during vitamin A deficiency, can be reversed by supplementation with retinoids (1). Furthermore, retinoids suppress squamous cell differentiation that occurs spontaneously or is induced by calcium ions in keratinocytes cultured from different human tissues $(1,3)$. Retinoids also suppress carcinogenesis in experimental animals including a variety of tissues such as the oral epithelium (4). Clinical studies have extended these observations to human patient care and demonstrated the efficacy of retinoids in suppressing oral premalignant lesions and the occurrence of second primary carcinomas in patients with head and neck cancer (5-7). These second primary tumors, which occur at an incidence rate of $2-3 \%$ per year, are the major cause of death after surgical resection of early-stage head and neck cancer (8). Retinoid treatment is however complicated by lack of activity in certain individuals and from marginal activity in advanced cancer. These observations clearly suggest that changes in the retinoid sensitivity may underlie tumor development.

Increasing experimental evidence suggests that vitamin A mediates its effects on the oral epithelial cells via its retinoic acid (RA) derivatives (9-11). All-trans and 9-cis RA act as ligands for the retinoid nuclear receptors (RARs, RXRs) (12). These receptors are ligand-dependent transcription factors, which bind to specific hormone response element (RARE, RXRE) leading to transactivation of specific target genes (13). Retinoic acids utilised by the cells are either taken up from the blood circulation, or formed from vitamin A via the subsequent action of various retinol and retinal dehydro- 
genases under the influence of intracellular retinol and retinoic acid binding proteins (14). Apart from uptake and synthesis, the amount of intracellular binding proteins and the activity of degrading enzymes also influence the intracellular levels and availability of retinoids for receptor interaction $(14,15)$.

The application of serum-free conditions has permitted the establishment of replicative cultures of normal oral keratinocytes, and the growth of both immortalized and malignant oral keratinocyte lines (16). Normal (NOK), SV40 T antigen-immortalized (SVpgC2a) and malignant (SqCC/Y1) human buccal keratinocytes model oral cancer development on the basis that they reflect the eventual acquisition of immortality, loss of p53 tumor suppressor function and gain of a tumorigenic phenotype $(16,17)$. Furthermore, these normal and transformed keratinocyte cell lines demonstrate keratin expression patterns similar to normal tissue, oral dysplasia and well-differentiated oral squamous cell carcinoma, respectively (18). The parallel examination of such lines thus provides an in vitro model composed of the various stages that lead to oral squamous carcinoma and which can be used to study expression of genes involved in retinoid action. On this basis, the current study analysed the expression pattern of 52 retinoid-related genes in $\mathrm{NOK}$, $\mathrm{SVpgC2} \mathrm{a}$ and $\mathrm{SqCC} / \mathrm{Y} 1$ using the Affymetrix micro-array chip technique. Notably, the use of one standardised culture condition provided an identical environment and exogenous influence on gene expression in the various cell types. The results thus obtained, suggest the possibility of multiple alterations in retinoid-related gene expression during oral carcinogenesis, including mechanisms that might reduce availability of retinoic acids and interfere with retinoidregulated signalling pathways.

\section{Materials and methods}

Processing of cell cultures. For processing of primary cell cultures, human buccal tissue was selected from non-smokeing, non-cancer patients undergoing maxillofacial surgery with approval from the Karolinska Institutet ethics committee. Primary keratinocyte lines were derived following incubation of tissue with $0.17 \%$ trypsin (Sigma) in phosphate buffered saline at $4^{\circ} \mathrm{C}$ for $18-24 \mathrm{~h}$, and subsequent seeding of keratinocyte aggregates and single cells at $5 \times 10^{3}$ cells $/ \mathrm{cm}^{2}$ onto fibronectin/ collagen-coated (Sigma) dishes in serum-free EMHA(epithelial medium with high levels of amino acids) (16). The immortal cell line SVpgC2a, derived by transfection and stable integration of the SV40T antigen into buccal keratinocytes, and a serum-free strain of the buccal carcinoma cell line SqCC/Y1 were also cultured in EMHA $(16,17)$. The normal keratinocyte lines were subjected to the transcript analysis in passage 2, the SVpgC2a line in passages 63 and 64 , and the SqCC/Y1 line in passages 125 and 128.

Preparation of labeled cRNA. Methods for cRNA preparation, the hybridisation reactions and data analysis were provided by the manufacturer (Affymetrix). Briefly, total RNA was prepared with RNeasy (Qiagen) from $3 \times 10^{6}$ cells of each type. Double-stranded cDNA was synthesized from $25 \mu \mathrm{g}$ total RNA using a cDNA synthesis kit (SuperScript Choice
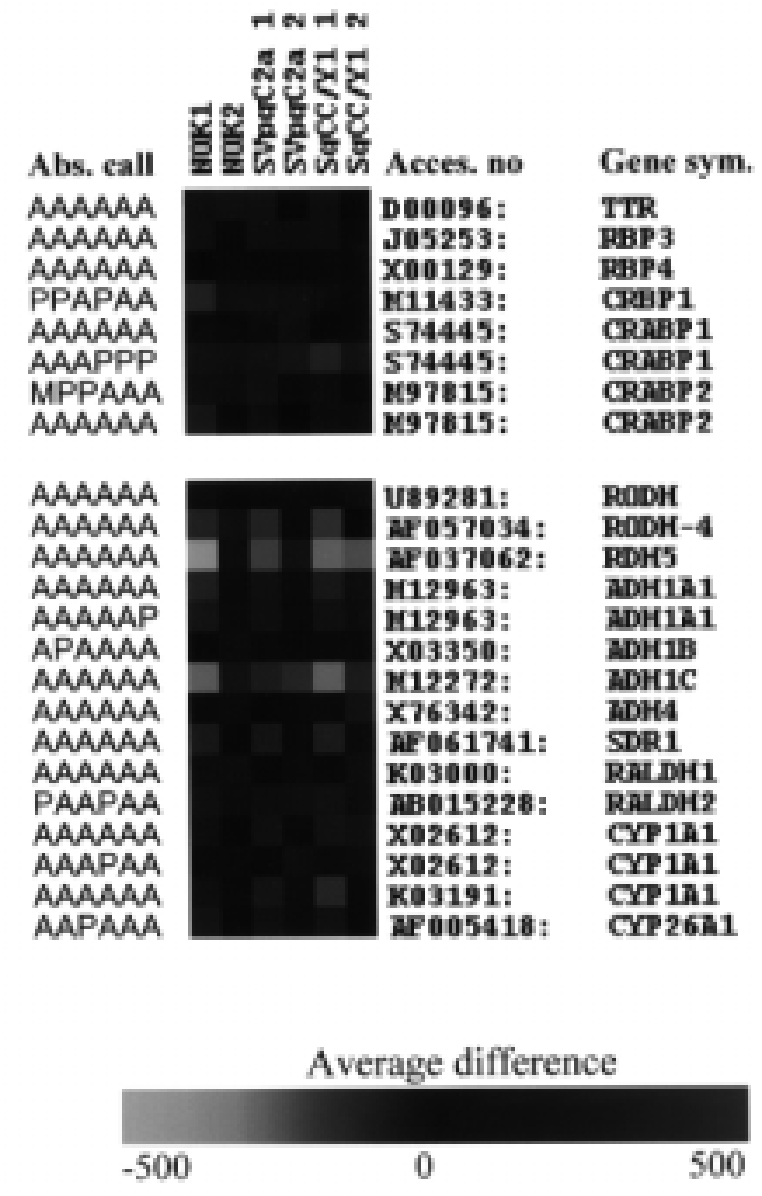

Figure 1. Expression pattern of genes involved in retinoid binding and metabolism as detected by Affymetrix gene array in NOK, SVpgC2a and SqCCY/1. The colour code used to express the average difference is shown below the gene panels. A, absent; P, present; M, marginally present.

system; Gibco BRL Life Technologies). Labeled cRNA was in vitro transcribed with $1.5 \mu \mathrm{g}$ cDNA as template incorporating biotinylated CTP and UTP (Enzo RNA Transcript Labeling Kit; Enzo Diagnostics). The cRNA was purified with RNeasy affinity columns (Qiagen) and subsequently

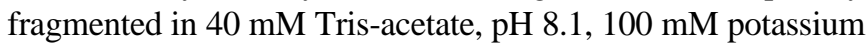
acetate, $30 \mathrm{mM}$ magnesium acetate at $94^{\circ} \mathrm{C}$ for $35 \mathrm{~min}$.

Array hybridization. cRNA $(10 \mu \mathrm{g})$ and samples for internal controls were hybridized to oligonucleotide arrays HG_U95A, (Affymetrix) at $45^{\circ} \mathrm{C}$ for $16 \mathrm{~h}$ according to the manufacturer's protocol. Arrays were washed under stringent conditions, stained with streptavidine-phycoerythrine (Molecular Probes), washed again and subsequently scanned at $570 \mathrm{~nm}$ using a Hewlett Packard Gene Array scanner. Obtained data were analyzed with GeneChip ${ }^{\circledR} 3.1$ software (Affymetrix). Visualisation of microarray data was performed by Cluster and TreeView software (downloaded from http://www.rana. lbl.gov). All chips were scaled to a target intensity of 250 for inter-array comparisons. The scale-factors for chip NOK (1), NOK (2), SVpgC2a (1), SVpgC2a (2), SqCC/Y1 (1) and $\mathrm{SqCC} / \mathrm{Y} 1$ (2) were $3.19,5.06,1.76,3.39,2.95$ and 3.83 respectively. The presence of individual genes was described by the absolute call (based on Pos/Neg Ratio, Positive Fraction 


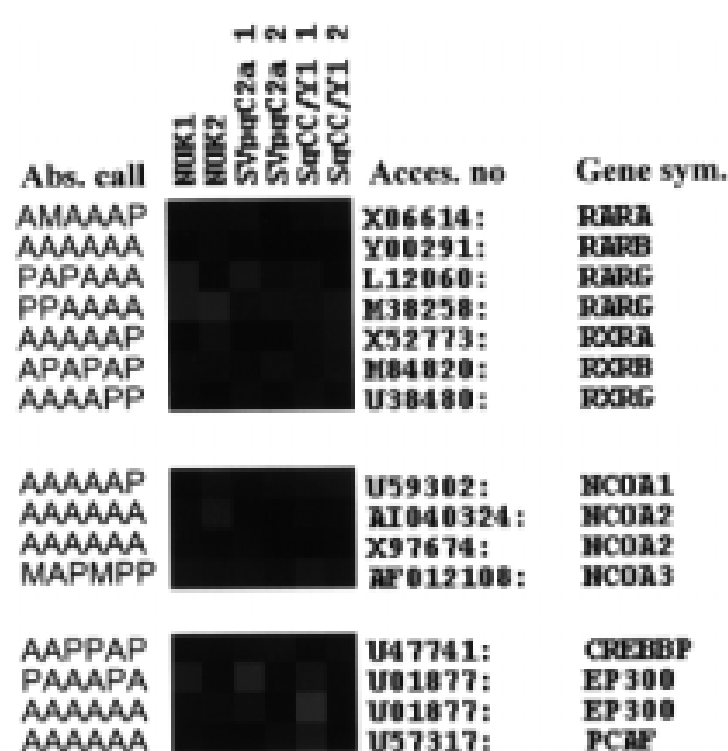

Average difference

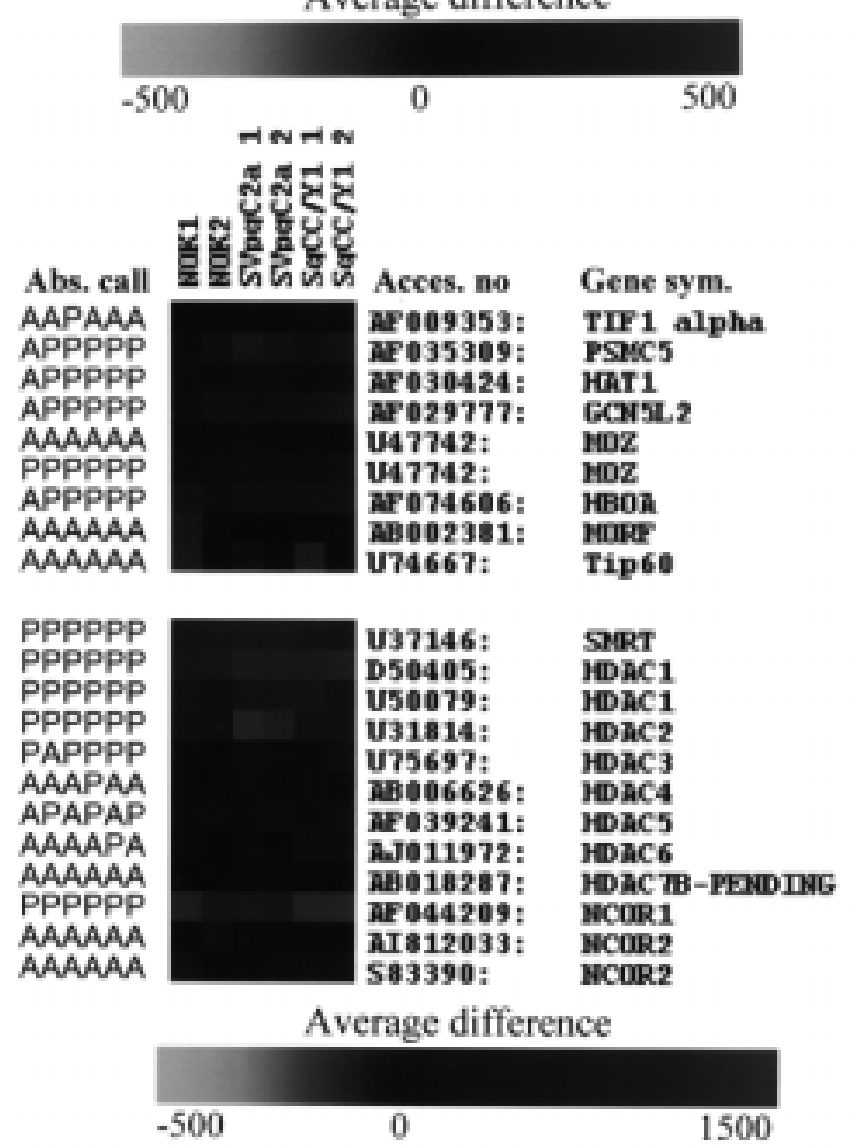

Figure 2. Expression pattern of genes involved in retinoid signalling as detected by Affymetrix gene array in NOK, SVpgC2a and SqCCY/1. The colour code used to express the average difference is shown below the gene panels. A, absent; P, present, M, marginally present.

and Log Average Ratio), while the changes in the expression levels were quantified by the average difference (for further details see GeneChip ${ }^{\circledR}$ 3.1 Expression Analysis Algorithm Tutorial). Gene expression was considered induced/repressed based on a two-fold change among samples. This limit is in agreement with other mammalian studies (19).
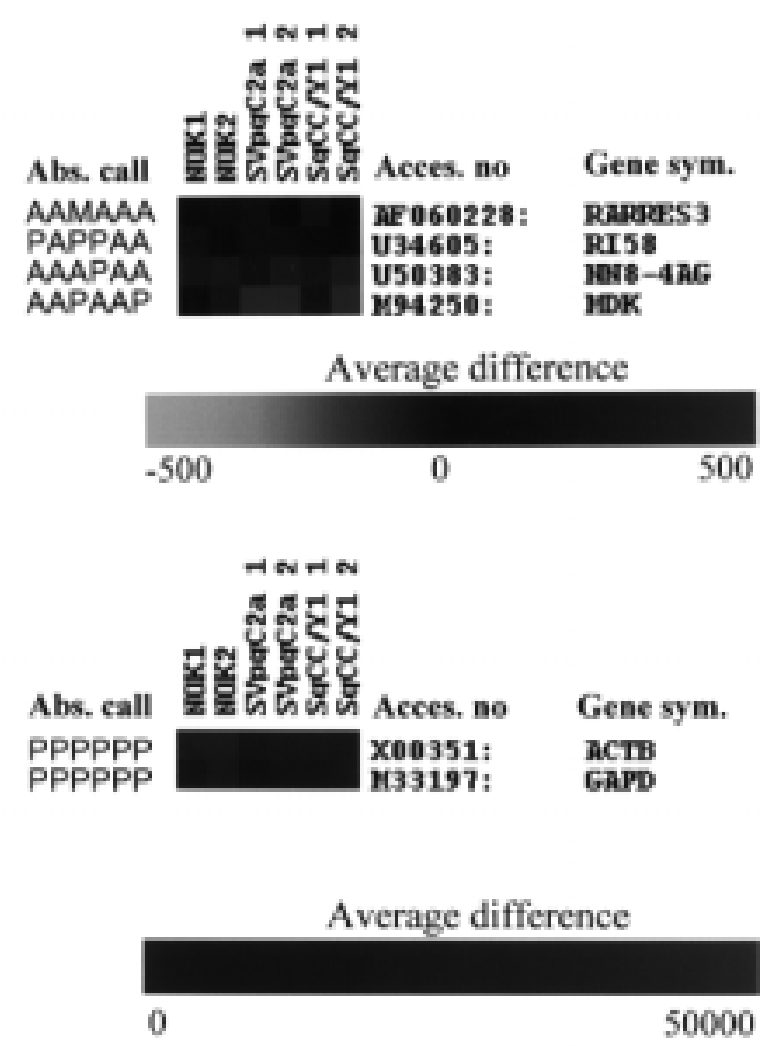

Figure 3. Expression pattern of four retinoid-responsive genes as detected by Affymetrix gene array. in $\mathrm{NOK}, \mathrm{SVpgC} 2 \mathrm{a}$ and $\mathrm{SqCCY} / 1$. The expression level of two 'house-keeping' genes, $B$-actin and glycerinaldehyde-3phosphate dehydrogenase, used as positive controls, is also shown. The colour code used to express the average difference is shown below the gene panels. A, absent; P, present, M, marginally present.

\section{Results}

Micro-array chips with oligonucleotides corresponding to 12558 human genes were hybridised with labelled cRNA prepared from NOK, immortalised (SVpgC2a) and malignant (SqCC/Y1) human oral keratinocytes to determine the expression level of retinoid-related genes. The results were from two separate experiments/hybridisations using different NOK lines and passages of SVpgC2a and SqCC/Y1. The total number of transcripts found in NOK, SVpgC2a and SqCC/Y1 in experiments 1 and 2 were 3830/3958, 4865/4467 and $3887 / 4533$, respectively. Altogether 52 genes involving different retinoid-related functions, i.e., binding/transport, metabolism, receptors, transcriptional activators/repressors, and finally, responsive genes, were examined. Probes for enzymes related to the retinol storage, e.g., lecithin-retinolacyltransferase (LRAT) or acyl-CoA-retinol acyltransferase, were absent on the chip. In contrast, probes for cellular retinoic acid binding proteins I and II, alcohol dehydrogenase 1 or RAR $\gamma$ were represented by two different probe sets. In total 22, 26 and 23 transcripts of the 52 genes were detected by the HG_U95A array in NOK, SVpgC2a and SqCC/Y 1 , respectively. The results are shown in Figs. 1-3 and discussed in detail below.

The transcripts of the retinol and RA binding proteins (CRBP-I, CRABP-II) responsible for storage and processing of retinol and RA (20) were all detected in the NOK lines, while no evidence was found for CRABP-I expression. 
Transcripts for CRBP-I and CRABP-II were absent, while that of CRABP-I was present, in at least one of the four hybridisations in $\mathrm{SVpgC2}$ a and $\mathrm{SqCC} / \mathrm{Y} 1$. All cell types were negative for retinol binding protein (RBP4), the transthyretin gene (TTR) and the interstitial retinoid binding protein (RBP3) involved in extracellular transport of retinoids (20). Thus, these three genes served as internal negative controls in the experiments.

Members of four enzyme families may participate in the conversion of retinol to retinoic acid (21). No transcripts were found for enzymes belonging to the short-chain dehydrogenase family responsible for the conversion of retinol to retinal, the first step in the synthesis of RA, such as retinol dehydrogenase (RODH), the microsomal $\mathrm{NAD}^{+}$dependent retinol dehydrogenase (RODH-4), short-chain dehydrogenase (SDR1), and 9-cis-/11-cis-retinol dehydrogenase (RDH5). Members of the alcohol dehydrogenase (ADH1A B C, ADH4) family, suggested also to participate in the retinol metabolism, were absent with the exception of ADH1B, which was detected in one of the NOK lines. There was no probe for the $\mathrm{ADH} 2$ enzyme on the chip.

Four enzymes have been suggested to participate in the conversion of retinal to RA, i.e., two alcohol dehydrogenases [retinal dehydrogenase (RALDH1=ALDH1) and RALDH2] and two cytochrome P450 types (P450 1A1 and 1A2). Message for RALDH1 was not detected. RALDH2 was detected in one of the two hybridisations in NOK and SVpgC2a, but not in $\mathrm{SqCC} / \mathrm{Y} 1$. CYP1A1 was not detected in any of the cell lines, and no probe was present for the CYP1A2 message on the chip.

The retinoic acid 4-hydroxylase (CYP26A1) responsible for the degradation of RA (22) was detected in only one of the analysis of SVpgC2a.

Analysis of members of the nuclear retinoid receptor family (12) indicated presence of RAR $\alpha$ in NOK and $\mathrm{SqCC} / \mathrm{Y} 1$, while no expression was detected in SVpgC2a. RARß was not detected in any of the cell lines. RAR $\gamma$ was detected in three of the four repeats in NOK, in one of the four repeats in the $S V p g C 2 a$, while its message was not detected in $\mathrm{SqCC} / \mathrm{Y} 1$. NOK and SVpgC2a showed transcripts of RXRß, whereas SqCC/Y1 showed presence of RXR $\alpha, \beta$ and $\gamma$ in at least one of the probe sets.

Members of the nuclear retinoid receptor family mediate their effects on the basal transcriptional machinery via interacting with other nuclear proteins (23). In the presence of the specific ligand they activate transcription via binding to various members of co-activators. Some of the major co-activators can be classified to the p160 family, including SRC-1/N-CoA1, GRIP-1/TIF2/N-CoA2 and ACTR/ pCIP/RAC3/AIB-1/N-CoA3; or to the co-integrators such as CBP (CREBBP), p300 and pCAF. Of the p160 family, $\mathrm{N}-\mathrm{CoA} 1$ was present in one of the analyses of $\mathrm{SqCC} / \mathrm{Y} 1$. $\mathrm{N}-\mathrm{CoA} 2$ was not detected in the cell types. N-CoA3 was marginally expressed or not detected in NOK, but it was present in $\mathrm{SVpgC2}$ a and in $\mathrm{SqCC} / \mathrm{Y} 1$. Of the co-integrators, CREBBP was not detected in NOK, but it was present in $\mathrm{SVpgC2a}$ and in one of the repeats in $\mathrm{SqCC} / \mathrm{Y} 1$. p300 was detected in one of the repeats in NOK and $\mathrm{SqCC} / \mathrm{Y} 1$ cell lines. pCAF was not detected in any of the cell lines. Of additional co-activator proteins, expression of TIF- $1 \alpha$ (transcriptional intermediary factor) was detected in one of the repeats in SVpgC2a, while PSMC5 was expressed by all cells. Coactivator family members often exhibit histone acetyltransferase activity and other histone acetyltransferases may also be transciptionally active (24). Histone acetyltransferases HAT1, Gcn5L2, MOZ and HBOA were expressed by each cell lines with no significant change in the expression level, except for Gcn5L2, which was elevated in SqCCY/1. In contrast, message for MORF or Tip60 histone acetyltransferases was not detected in any of the cell lines.

In the absence of specific ligand, RARs repress basal transcription by binding to promoters of target genes and by interaction with two co-repressors $\mathrm{N}$-Cor (nuclear receptor co-repressor) and SMRT (silencing mediator of retinoid and thyroid hormone receptor) $(25,26)$. Analogous to the co-activator complexes, these co-repressors recruit histone deacetylases (HDACs) (27). While SMRT was shown to recruit class I HDACs, such as HDAC1, HDAC2, HDAC3 and HDAC8, N-Cor reacts with class II HDACs, such as HDAC4, HDAC5 and HDAC7 (28). N-Cor1 and SMRT were expressed in each cell line, while no message for N-Cor2 was detected. HDAC 1, 2, 3 and 5 were expressed in each cell line, HDAC2 showing a higher expression in $\mathrm{SVpgC2}$ a relative to NOK. HDAC4 was detected in one of the repeats in $\mathrm{SVpgC2a}$, HDAC6 was detected in one repeats in ScCC/Y1, while HDAC7 was not expressed. There was no probe for HDAC8 on the chip.

Finally, expression of some retinoid-responsive genes was analysed. RARRES3 (or TIG3), the retinoic acid receptor responder 3 (29), was marginally expressed in one of the SVpgC2a preparations. mRNA of the NN8-4AG (30) was detected in one of the two SVpgC2a repetitions. The retinoic acid and interferon inducible protein, RI58 (31) was detected in one of the analyses of NOK and in both analyses of $\mathrm{SVpgC2a}$. Finally, the retinoic acid inducible factor midkine (32) was not detected in NOK but it was detected at high levels in one analysis of each of the transformed lines.

\section{Discussion}

In this study, the expression pattern of 52 retinoid-related genes was studied under identical serum-free conditions using an in vitro model of oral carcinogenesis that includes normal, immortalized and malignant cells. The existence of transcripts from genes coding for retinoid-related functions were analyzed based on the concept that the responsiveness of oral keratinocytes may decrease during malignant transformation (33). As discussed separately for various retinoidrelated genes, including binding/transport, metabolism, receptors, transcriptional activators/repressors and responsive genes, the overall results supported an association between impaired retinoid sensitivity and oral carcinogenesis.

To maintain solubility, retinoids must be bound to proteins in an aqueous environment. Hepatic proteins have been reported to participate in the extra-cellular transport of retinoids (20). As expected for extra-hepatic tissues, the oral keratinocyte lines were negative for retinol binding protein and transthyretin, and furthermore, the cells were negative for the interstitial retinoid binding protein that is typically expressed in the eye (20). 
The uptake and the storage of retinoids within the cells are mediated by various cellular retinoid binding proteins including CRBP-I, CRABP-I and II. CRBP-I facilitates retinol uptake, delivers retinol to LRAT for esterification and storage, and interacts with several enzymes that metabolise retinol or retinal, providing the retinol and retinal substrate for their action (20). Of the cellular retinoid-binding proteins, CRBP-I manifests the widest distribution, expressed in most retinoid-sensitive tissues. Accordingly, CRBP-I was clearly detected in NOK, however, it was not detected in $\mathrm{SqCC} / \mathrm{Y} 1$. CRBP-1-deficient mice have decreased capacity to store incoming retinol and to maintain retinyl ester stores, and show enhanced sensitivity to vitamin A deficiency (34) suggesting that loss of the CRBP-1 function might have the same impact on carcinoma cells. In agreement, loss of CRBP-1 is an early event during transformation of breast epithelium, a known retinoid-sensitive tissue (35).

Though the two cellular RA binding proteins possess similar RA-binding affinities, they display distinct pattern of expression across cells and developmental stages, and serve specific and distinct roles in RA biology $(36,37)$. Elevated CRABP-I expression in F9 teratocarcinoma cells enhanced formation of polar metabolites from RA and blocked RAinduced differentiation (38). Accordingly, increased CRABP-I expression, as noted in $\mathrm{SqCC} / \mathrm{Y} 1$ relative to the other cell types, may lead to decreased availability of RA. In contrast, CRABP-II sensitises cells to RA and facilitates transfer of RA to RAR via direct interaction with the nuclear receptor $(15,39)$. CRABP-II was present in NOK but undetected in $\mathrm{SqCC} / \mathrm{Y} 1$, whereas CRABP-I was undetected in NOK but present in SqCC/Y1. High and low levels of CRABP-II were previously reported for normal keratinocytes and $\mathrm{SqCC} / \mathrm{Y} 1$, respectively, using semi-quantitative RT-PCR (9). Overall, the expression pattern for CRABP proteins implied decreased RA sensitivity in SqCC/Y1 as compared to NOK.

The pathway for conversion of retinol to RA involves oxidation of retinol to retinal followed by oxidation of retinal to retinoic acid, either in free or in CRBP-bound form. Enzymes involved in retinol and retinal metabolism belong to four distinct families; i.e. alcohol dehydrogense, short-chain dehydrogenase/reductase, aldehyde reductase and cytochrome P450 (21). The microarray results implied low or absent, i.e., undetected, expression of these enzymes in the cell lines, with the exception of RALDH2 (41), that was detected in one of the two probe sets in NOK and SVpgC2a. The CYP26A1 (retinoic acid 4-hydroxylase), an enzyme responsible for the catabolism of retinoic acid (22), was detected in only one of the assessments of SVpgC2a. Elevated enzyme levels of this enzyme were previously reported in head and neck cancers potentially leading to increased retinoid metabolism and decreased retinoid availability (42).

The spatio-temporal distribution of various nuclear retinoic acid receptors during embryonic development and tissue-specific expression patterns suggest differential roles of these receptors in retinoid-regulated signalling pathways (43). RAR $\alpha$ and RAR $\gamma$ but not RARß, was detected in NOK in agreement with previous studies in keratinocytes cultured from different regions of normal oral mucosa (44). Message for RARß was not detected in $\mathrm{SVpgC2a}$ or $\mathrm{SqCC} / \mathrm{Y} 1$, whereas message for $\mathrm{RAR} \alpha$ was detected in $\mathrm{SqCC} / \mathrm{Y} 1$ but not in
SVpgC2a. In agreement, presence of $\operatorname{RAR} \alpha$ and lack of $\mathrm{RAR} 3$ expression was previously reported for SqCC/Y1 (9). Only one of the four repeats for RAR $\gamma$ was positive in $\mathrm{SVpgC2a}$ and none of the repeats were positive in SqCC/Y1. The transcript levels for RAR $\gamma$ in SqCCY1 are likely below the detection limit of the micro-array, since SqCC/Y1 was previously shown to express low levels of RAR $\gamma$ and to respond to $\mathrm{RAR} \gamma$ specific compounds (9). SqCC/Y1 transfected with RAR $\gamma$ show increased sensitivity to growth inhibition by RA while antisense-transfection causes the opposite effect (10). Furthermore, the expression level of RAR $\gamma$ correlates to RA sensitivity of head and neck squamous carcinoma cell lines (45). In this regard, the low RAR $\gamma$ expression levels of $\mathrm{SVpgC2}$ a and $\mathrm{SqCC} / \mathrm{Y} 1$ as detected by micro-array indicate that both lines may be relatively insensitive to retinoid actions.

RXRs serve as dimerisation partners for the RAR receptors. Unlike the RAR receptors that are stimulated by both alltrans and 9-cis RA, the RXRs are selectively stimulated by 9-cis RA (12). The micro-array results suggested increased expression of RXRs in the malignant state since RXR $\alpha$ and RXR $\gamma$ was selectively expressed in SqCC/Y1, whereas $\mathrm{RXR} \beta$ was detected in one repetition of all cell types. In agreement, increased level of RXRs was previously reported for oral carcinomas (46). The role of RXRs for retinoid signalling in oral keratinocytes remains unclear. However, stimulation of RXR might inhibit the growth inhibitory signals mediated by the RAR receptors, since NOK are growth inhibited by nano-molar levels of all-trans RA and $\mu$ molar levels of 9-cis RA (unpublished observation). In this regard, increased RXR expression in the malignant state might again lead to decreased retinoid sensitivity.

Various nuclear proteins interact with the retinoid receptors thereby mediating repression or activation of specific target genes. In absence or presence of receptor ligand, complexes of corepressors or coactivators mediate histone deacetylation or acetylation, respectively, and such reversible acetylation is thought to regulate protein to DNA interactions and transcription $(27,47)$. The co-activator complex members, ACTR/pCIP/RAC3/AIB-1/N-CoA3 (48), its binding partner CBP/p300 (49), PSMC5 (50), and that of the histone acetyltransferases HAT1, Gcn5L2 belonging to the GNAT superfamily, and MOZ and HBOA belonging the MYST family (24) were detected at similar expression levels in all cell types with the exception of Gen5L2, the message of which was elevated in SqCC/Y1. The co-repressor complex members, SMRT, Ncor-1, and the histone deacetylase 1,2,3 and 5 were detected at similar expression level in all cell types except for higher levels of HDAC2 in SVpgC2a. The expression levels of HDAC4 and 6 may be close to the detection limit of the micro-array assay since their transcripts were detected in only one probe set. Though some reports suggest that proteins such as CBP/p300 or MOZ may act to suppress cancer development $(51,52)$, the current results in the oral keratinocyte lines did not imply that transformation induces significant alteration in the expression of the nuclear co-activators and co-repressors.

Finally, expression of certain retinoid-regulated genes was implicated for oral epithelium from positive detection in at least one of the analysis of the respective cell types (Fig. 3). 
The cellular function of NN8-4AG (30) is unknown, whereas RARRES or TIG3 (29) is considered a tumor-suppressor that is down-regulated in B-cell lymphoid leukemia progression (53). The retinoic acid and interferon inducible protein (RI58) has a known involvement in myeloid differentiation (31). Midkine is a heparin-binding growth/differentiation regulating factor that promotes neuronal growth $(54,55)$. Its expression in human epidermal keratinocytes was also associated to promoted growth (56). On the basis of elevated serum levels in cancer patients (57), the possibility of selective expression in the immortal and malignant states indicates an association also to the development of oral squamous carcinomas. As for the other retinoid-related functions investigated in this study, future assessments of tissue-specificity or variability among cell lines should consider methods of even higher sensitivity than microarray, e.g., quantitative RT-PCR (58), in order to more accurately determine presence or absence of gene expression in quantitative terms.

In conclusion, application of the micro-array chip technology allowed a simultaneous expression analysis of a large number of retinoid-related genes in a three-step progression model of oral carcinogenesis. The expression patterns under identical growth conditions indicated alterations in regards to the metabolism and signalling of retinoids, ultimately implicating decreased retinoid sensitivity during cell transformation. The noted retinoid-related changes included those that could affect binding, storage, synthesis and signalling, and finally, genes that are regulated by retinoids. The overall analyses thus suggest a variety of mechanisms for normal and disturbed retinoid actions in oral epithelium, some of which are likely to be testable in future assessments of growth and cell death regulation in cultured normal and transformed oral keratinocytes.

\section{Acknowledgements}

This work was supported by the Swedish Cancer Society, the Smokeless Tobacco Research Council in USA, the Swedish National Board of Laboratory Animals, the Swedish Fund for Research Without Animal Experiments, Swedish Match and Preem Environment Fund.

\section{References}

1. Lotan R: Retinoids and squamous cell differentiation. In: Retinoids in Oncology. Hong WK and Lotan R (eds). Marcel Dekker, New York, pp43-72, 1993.

2. Gudas LJ, Sporn MB and Roberts AB: Cellular biology and biochemistry of retinoids. In: The Retinoids. Sporn MB, Roberts AB and Goodman DS (eds). Raven Press, New York, pp443-520, 1994.

3. Jetten AM, Nervi C and Vollberg TM: Control of squamous differentiation in tracheobronchial and epidermal epithelial cells: role of retinoids. J Natl Cancer Inst Monogr 13: 93-100, 1992.

4. Moon RC and Mehta RG: Retinoid inhibition of experimental carcinogenesis. In: Chemistry and Biology of Synthetic Retinoids. Dawson MI and Okamura WH (eds). CRC Press, Boca Raton, FL, pp501-518, 1990.

5. Hong WK, Endicott J, Itri L, et al: 13-cis retinoic acid in the treatment of oral leukoplakia. N Engl J Med 315: 1501-1505, 1986.

6. Chiesa F, Tradati N, Marazza M, et al: Prevention of local relapses and new localisations of oral leukoplakia with synthetic retinoid fenretinide (4-HPR). Preliminary results. Oral Oncol Eur J Cancer 28B: 97-102, 1992.
7. Hong WK, Lippman SM, Itri L, et al: Prevention of secondary primary tumors with osotretinoin in squamous cell carcinoma of the head and neck. N Engl J Med 323: 795-801, 1990.

8. Snow GB: Follow-up in patients treated for head and neck cancer: how frequent, how thorough and for how long? Eur $\mathrm{J}$ Cancer 28: 315-316, 1992.

9. Zou CP, Clifford JL, Xu XC, et al: Modulation by retinoic acid (RA) of squamous differentiation, cellular RA-binding proteins, and nuclear RA receptors in human head and neck squamous cell carcinoma cell lines. Cancer Res 54: 5479-5487, 1994.

10. Oridate N, Esumi N, Lotan D, et al: Implication of retinoic acid receptor $\gamma$ in squamous differentiation and response to retinoic acid in head and neck SqCC/Y1 squamous carcinoma cells. Oncogene 12: 2019-2028, 1996.

11. Hayashi K, Yokozaki H, Naka K, Yasui W, Lotan R and Tahara E: Overexpression of retinoic acid receptor $\beta$ induces growth arrest and apoptosis in oral cancer cell lines. Jpn J Cancer Res 92: 42-50, 2001.

12. Chambon P: The retinoid signaling pathway: molecular and genetic analyses. Semin Cell Biol 5: 115-125, 1994.

13. Rastinejad F, Perlmann T, Evans RM and Sigler PB: Structural determinants of nuclear receptor assembly on DNA direct repeats. Nature 375: 203-211, 1995.

14. Napoli JL: Interactions of retinoid binding proteins and enzymes in retinoid metabolism. Biochim Biophys Acta 1440: 139-162, 1999.

15. Delva L, Bastie JN, Rochette-Egly C, et al: Physical and functional interactions between cellular retinoic acid binding protein II and the retinoic acid-dependent nuclear complex. Mol Cell Biol 19: 7158-7167, 1999.

16. Grafström RC: Human oral epithelium. In: Culture of Epithelial Cells. Freshney RI and Freshney MG (eds). John Wiley and Sons, Inc., New York, pp195-255, 2002.

17. Kulkarni PS, Sundqvist K, Betsholtz C, et al: Characterization of human buccal keratinocytes transfected with the simian virus 40 T-antigen gene. Carcinogenesis 16: 2515-2521, 1995.

18. Hanson A, Bloor BK, Haig Y, Morgan PR, Ekstrand J and Grafström RC: Expression of keratins in normal, immortalized and malignant oral epithelia in organotypic culture. Oral Oncol 5: 419-430, 2001.

19. Fambrough D, McClure K, Kazlauskas A and Lander ES: Diverse signaling pathways activated by growth factor receptors induce broadly overlapping, rather than independent, sets of genes. Cell 97: 727-741, 1999.

20. Gottesman ME, Quadro L and Blaner WS: Studies on vitamin A metabolism in mouse model systems. Bioessays 23: 409-419, 2001.

21. Duester G: Involvement of alcohol dehydrogenase, short-chain dehydrogenase/reductase, aldehyde dehydrogenase, and cytochrome $\mathrm{p} 450$ in the control of retinoid signaling by activation of retinoic acid synthesis. Biochemistry 35: 12221-12227, 1996.

22. White JA, Beckett-Jones B, Guo YD, et al: cDNA cloning of human retinoic acid-metabolising enzyme (hP450RAI) identifies a novel family of cytochromes P450 (CYP26). J Biol Chem 272: 18702-18708, 1997.

23. Freedman LP: Increasing the complexity of coactivation in nuclear receptor signaling. Cell 97: 5-8, 1999.

24. Sterner DE and Berger SL: Acetylation of histones and transcription-related factors. Microbiol Mol Biol Rev 64: 435459, 2000.

25. Chen JD and Evans RM: A transcriptional co-repressor that interacts with nuclear hormone receptors. Nature 377: 454-457, 1955.

26. Kurokawa R, Söderstrom M, Hörlein A, et al: Polarity-specific activities of retinoic acid receptors determined by a co-repressor. Nature 377: 451-454, 1995.

27. Pazin JM and Kadonaga JT: What's up and down with histone deacetylation and transcription? Cell 89: 325-328, 1997.

28. Bertos NR, Wang HA and Yang X: Class II histone deacetylases: structure, function, and regulation. Biochem Cell Biol 79: 243-252, 2001.

29. Di Sepio D, Ghosn C, Eckert RL, et al: Identification and characterization of a retinoid-induced class II tumor suppressor/ growth regulatory gene. Proc Natl Acad Sci USA 95: 14811$14815,1998$.

30. Shago $\mathrm{M}$ and Giguere $\mathrm{V}$ : Isolation of a novel retinoic acidresponsive gene by selection of genomic fragments derived from CpG-island-enriched DNA. Mol Cell Biol 16: 4337-4348, 1996. 
31. Niikura T, Hirata R and Weil SC: A novel interferon-inducible gene expressed during myeloid differentiation. Blood Cell Mol Dis 23: 337-349, 1997.

32. Uehara K, Matsubara S, Kadomatsu K, Tsutsui J and Muramatsu T: Genomic structure of human midkine (MK), a retinoic acid-responsive growth/differentiation factor. J Biochem 111: 563-567, 1992.

33. Hong WK and Itri LM: Retinoids and human cancer. In: The Retinoids. Sporn MB, Roberts AB and Goodman DS (eds). Raven Press, New York, pp597-630, 1994.

34. Ghyselinck NB, Båvik C, Sapin V, et al: Cellular retinol-binding protein I is essential for vitamin A homeostasis. EMBO J 18: 4903-4914, 1999

35. Kuppumbatti YS, Bleiweiss IJ, Mandeli JP, Waxman S and Mira YL: Cellular retinol-binding protein expression and breast cancer. J Natl Cancer Inst 92: 475-480, 2000.

36. Dong D, Ruuska SE, Levinthal DJ and Noy N: Distinct roles for cellular retinoic acid-binding proteins I and II in regulating signaling by retinoic acid. J Biol Chem 274: 23695-23698, 1999.

37. Maden M: Distribution of cellular retinoic acid-binding proteins I and II in the chick embryo and their relationship to teratogenesis. Teratology 50: 294-301, 1994.

38. Boylan JF and Gudas LJ: Overexpression of the cellular retinoic acid binding protein-I (CRABP-I) results in a reduction in differentiation-specific gene expression in F9 teratocarcinoma cells. J Cell Biol 112: 965-979, 1991.

39. Jing Y, Waxman S and Mira-y-Lopez R: The cellular retinoic acid binding protein II is a positive regulator of retinoic acid signaling in breast cancer cells. Cancer Res 57: 1688-1692, 1997.

40. Siegenthaler G, Tomatis I, Chatellard-Gruaz D, Jaconi S, Eriksson U and Saurat JH: Expression of CRABP-I and -II in human epidermal cells. Alterations of relative protein amounts is linked to the state of differentiation. Biochem J 287: 383-389, 1992.

41. Neiderreihter K, Subbarayan V, Dolle P and Chambon P: Embryonic retinoic acid synthesis is essential for early mouse post-implantation development. Nat Genet 21: 444-448, 1999.

42. Klaassen I, Brakenhoff RH, Smeets SJ, Snow GB and Braakhuis BJ: Enhanced turnover of all-trans-retinoic acid and increased formation of polar metabolites in head and neck squamous cell carcinoma lines compared with normal oral keratinocytes. Clin Cancer Res 7: 1017-1025, 2001.

43. Zile MH: Function of vitamin A in vertebrate embryonic development. J Nutr 131: 705-708, 2001.

44. Crowe DL, Hu L, Gudas LJ and Rheinwald JG: Variable expression of retinoic acid receptor (RAR-beta) mRNA in human oral epidermal keratinocytes: relation to keratin 19 expression and keratinization potential. Differentiation 48: 199-208, 1991.
45. Klaassen I, Brakenhoff RH, Smeets SJ, Snow GB and Braakhuis BJ: Expression of retinoic receptor gamma correlates with retinoic acid sensitivity and metabolism in head and neck squamous carcinoma cell lines. Int J Cancer 92: 661$665,2001$.

46. Sherman JA and Partridge M: Expression of retinoic acid receptors in normal, dysplastic and malignant oral epithelia. $\mathrm{Br} \mathbf{J}$ Oral Maxillofac Surg 35: 260-266, 1997.

47. Nagy L, Kao HY, Love JD, et al: Mechanism of corepressor binding and release from nuclear hormone receptors. Genes Dev 13: 3209-3216, 1999.

48. Chen H, Lin RJ, Schiltz RL, et al: Nuclear receptor coactivator ACTR is a novel histone acyltransferase and forms a multimeric activation complex with pCAF and CBP/p300. Cell 90: 569-580, 1997.

49. Bannister AJ and Kouzarides T: The CBP co-activator is histone actyltransferase. Nature 384: 641-643, 1996.

50. Lee JW, Ryan F, Swaffield JC, Johnston SA and Moor D: Identification of thyroid-hormone receptor with a conserved transcription mediator. Nature 374: 91-94, 1995.

51. Gayther SA, Batley SJ, Linger L, et al: Mutations truncating the EP300 acetylase in human cancers. Nat Genet 24: 300-303, 2000.

52. Giles RH, Peters DJM and Breuning MH: Conjunction dysfunction: CBP/p300 in human disease. Trends Genet 14: 178-183, 1998.

53. Casanova B, De la Fuente MT, Garcia-Gila M, et al: The class II tumor-suppressor gene RARRES3 is expressed in B cell lymphocytic leukemias and down-regulated with disease progression. Leukemia 15: 1521-1526, 2001.

54. Muramatsu $\mathrm{H}$ and Muramatsu $\mathrm{T}$ : Purification of recombinant midkine and examination of its biological activities: functional comparison of new heparin-binding factors. Biochem Biophys Res Commun 177: 652-658, 1991.

55. Muramatsu H, Shirahama H, Yonezawa S, Maruta $H$ and Muramatsu T: Midkine, a retinoic acid-inducible growth/ differentiation factor: immunological evidence for the function and distribution. Dev Biol 159: 392-402, 1993.

56. Inazumi T, Tajima S, Nishikawa T, Kadomatsu K, Muramatsu H and Muramatsu T: Expression of the retinoid-inducible polypeptide, midkine, in human epidermal keratinocytes. Arch Dermatol Res 289: 471-475, 1997.

57. Ikematsu S, Yano A, Aridome K, et al: Serum midkine levels are increased in patients with various types of carcinomas. Br J Cancer 83: 701-706, 2000.

58. Crawford EL, Peters GJ, Noordhuis P, et al: Reproducible gene expression measurement among multiple laboratories obtained in a blinded study using standardized RT (StaRT)-PCR. Mol Diagn 6: 217-225, 2001. 